\title{
UN ÉTAT DE SECOND DEGRÉ : LE DOCUMENT PHOTOGRAPHIQUE À L'ÉPREUVE DE L'ART CONTEMPORAIN
}

\begin{abstract}
Alexandre Streitberger ${ }^{1}$
Le but de cet essai est de mettre en relation deux pratiques récurrentes dans l'art photographique contemporain : d'une part, l'emploi de la photographie documentaire ou bien du document photographique comme matériau de base et, d'autre part, ce que j'appelle "La photographie au second degré" en paraphrasant le sous-titre de l'ouvrage bien connu de Gérard Genette, Palimpsestes. La littérature au second degré. Bien entendu, l'intention n'est pas de transférer la taxonomie de Genette au champ de la photographie, les différences catégoriques entre les deux média - la littérature et la photographie - ne permettant qu'un rapprochement très prudent. Cela dit, il nous semble toutefois opportun, pour une première tentative de définition, d'évoquer la structure de duplicité qui caractérise selon Genette le palimpseste "où l'on voit, sur le même parchemin, un texte se superposer à un autre qu'il ne
\end{abstract}

1 Professeur au Département d'archéologie et d'histoire de l'art de l'UCL.

Recherches en communication, $\mathrm{n}^{\circ} 27$ (2007). 
dissimule pas tout à fait, mais qu'il laisse voir par transparence"1. Par analogie, une photographie au second degré serait une photographie qui dérive d'autres photographies préexistantes pour créer une nouvelle image photographique. Il s'agit donc d'une image parasitaire ou, plus précisément, d'une image différée qui dégage un potentiel de réflexion, d'une image qui redouble et transforme en même temps l'apparence, le contexte et le message de la photo originale pour la mettre à l'épreuve.

Les ancêtres de la photographie au second degré sont évidemment les procédés du photocollage et du photomontage qui, en art, remontent aux expériences des avant-gardes des années 1920. Voici, le principe fondamental de cette méthode artistique décrit par François Soulages sous l'étiquette d'esthétique du transfert: "le concept de transfert désigne le déplacement d'une ou plusieurs photos d'un premier espace relevant soit du sans-art [...], soit de l'art, vers un deuxième espace qualifié d'objet artistique - comme un tableau, une sculpture, une autre photo, etc. En changeant de lieu, la photo peut changer de nature et changer la nature du nouveau lieu : métamorphose de l'art"2. Cette définition convient parfaitement à la photographie au second degré, car il s'agit bel et bien d'un procédé de transfert impliquant la décontextualisation et la recontextualisation de l'image.

Pour l'installation Amplification, par exemple, réalisée dans l'église San Stae à Venise en 1995, l'artiste suisse Christian Marclay a choisi six images provenant d'albums de famille qu'il avait trouvés au marché aux puces ${ }^{3}$. Agrandis sur de grands voiles transparents, les personnages surdimensionnés se superposent l'un à l'autre et deviennent ainsi des apparitions évanescentes de fantômes. Perdues en tant que souvenirs de famille, les images resurgissent dans le lieu de culte en tant qu'images de dévotion, avant de se perdre à nouveau. En plus, l'évocation d'une apothéose des souvenirs perdus, suggérée par l'échelle surdimensionnée des agrandissements dans la nef, se dévoile comme métaphore des mécanismes peu fiables de notre mémoire où les souvenirs disparaissent et réapparaissent, se superposent et s'évanouissent. Tout en maintenant l'intégrité de l'image, Marclay la transforme en image poly-

1 G. GENETTE, Palimpsestes. La littérature au second degré, Paris, Seuil, 1982, p. 451.

2 F. SOULAGES, Esthétique de la photographie, Paris, Armand Colin, 2005, p. 247.

3 C. MARClay, Amplification, Biennale di Venezia 1995, Svizzera, Chiesa di San Stae, Baden, Éditions Lars Müller, 1995. 
valente, invitant le spectateur à une réflexion sur la mémoire, l'usage cultuel de l'image et les fonctions de la photo.

Pour distinguer ce genre de manipulation photographique du photocollage et du photomontage, il faut constater que ces derniers sont fondés sur un principe esthétique où le matériel photographique est découpé, fragmenté, decontextualisé et recomposé pour constituer un tableau. C'est seulement une fois que la composition est achevée que cette œuvre unique peut - mais ne doit pas - être reproduite photomécaniquement. Le principe de la photographie au second degré, par contre, est la transformation d'une photo par la reproduction de l'original, une sorte de re-reproduction. Plus qu'une réorganisation esthétique d'éléments photographiques, il s'agit donc d'un procédé qui touche à l'essentiel de la photographie en tant que médium de reproduction. En fait, la photographie au second degré, nous le verrons, s'engage dans une rhétorique de la duplication, qui la rapproche de la structure du mythe comme elle a été définie par Roland Barthes pour, enfin, saper les mythes associés au médium de la photographie.

\section{Du document photographique à la photographie documen- taire et l'inverse...}

Historiquement, le nouvel intérêt pour la photographie de la part des artistes coïncide avec la redécouverte de la photographie documentaire aux Etats-Unis au cours des années 1960.

Notamment le département des Photographies du Museum of Modern Art à New York y consacre plusieurs expositions promouvant Walker Evans comme figure-phare du mouvement, sa rétrospective de 1971 marquant le comble de cette renaissance. Concurremment le Pop art et puis l'art conceptuel découvrent le document photographique comme sujet (le Pop) et comme signe de témoignage (Art Conceptuel). Pour décortiquer le rapport complexe qu'entretient la photographie documentaire avec l'art photographique, ou plus précisément avec l'art utilisant la photographie comme matériau artistique, il est indispensable de mettre en relief la distinction entre document photographique et photographie documentaire, voire style documentaire. Or, depuis l'invention du médium, le document photographique est apprécié pour sa valeur scientifique ou archivale en tant qu' apport d'informations, de 
témoignage ou de preuve. Partant de l'idée que "l'art n'est jamais un document, mais [...] peut en adopter le style"1, Walker Evans invente dans les années 1930 ce qu'il appelle "le style documentaire", qui s'appuie sur deux piliers : d'abord l'idée du document comme forme dont les critères sont parfaitement décrits par le photographe lui-même en 1964 : "[Ce style] a de la pureté, et une certaine sévérité, de la rigueur, de la simplicité, un caractère direct, de la clarté"'2. Ensuite, l'idée de la documentation humanitaire dans la tradition de la Farm Security Administration, comme le souligne Beaumont Newhall en 1949 lorsqu'il la définit par la volonté "de persuader et de convaincre", d' "éveiller la conscience sociale" 3 .

Je ne veux pas m'étendre sur le rapport des artistes américains des années 1960 avec Walker Evans qui a été étudié à maintes reprises ${ }^{4}$. Cependant, il vaut la peine de prendre en considération une œuvre de Dan Graham afin d'illustrer brièvement les confusions engendrées par cette distinction entre document photographique et style documentaire évoquée tantôt.

Dans son photo-texte Homes for America datant de 1966, Graham met en évidence la stérilité anonyme des cités pavillonnaires américaines, construites en unités modulaires préfabriquées et standardisées. Les photographies accompagnant le texte illustrent les principes fondamentaux de ces pavillons de banlieue qui, selon l'auteur, correspondent parfaitement aux principes esthétiques du minimal art : la répétition, le dépouillement de la forme, le cube comme élément de base, la disposition sérielle. Les images utilisées par l'artiste rappellent ostensiblement les photos d'architecture prises par Evans quelques décennies auparavant. Par conséquent, il n'est pas étonnant que dans l'article finalement publié dans l'Arts Magazine en 1967, les photos de Graham aient été remplacées par une seule image de Walker Evans. En fait, à première vue la décision des éditeurs semble tout à fait pertinente si l'on se souvient que Walker Evans élève la photographie documentaire au niveau d'un style artistique, étant, de surcroît, susceptible d'éveiller la conscience sociale.

1 Walker Evans dans : O. LUGON, Le style documentaire. D'August Sander à Walker Evans, Paris, Macula, 2001, p. 18.

2 Ibid., p. 13.

3 Ibid., p. 10.

4 Voir par exemple : P. GALASSI (sous la dir. de), Walker Evans \& Company, Cat., Museum of Modern Art New York, 2000. 
Pourtant, les choses sont bien plus compliquées. L'objectif de Graham n'était pas l'inauguration d'un nouveau style à visée humanitaire, mais l'emploi de la photographie en tant que document dans le sens d'un apport d'informations, justement pour s'opposer au modernisme prônant l'autoréflexion de la peinture par l'exploitation des qualités formelles propres au médium. Lorsque Graham constate : "I wanted to show that Minimalism was related to a real social situation that could be documented"1, il est aux antipodes du style documentaire vanté par toute une génération de photographes et de curateurs dans les années 1960. Utilisant une camera Kodak et du matériel photographique à bon marché, il ne s'intéresse qu'à la valeur de preuve, de témoignage de la photo qui, réduite de nouveau au document photographique, doit rendre crédible le fait que l'art est une question d'information, non un exercice de style. Cependant, l'histoire de l'art conceptuel nous a fait comprendre que la photographie n'est ni neutre ni transparente et aucunement immune du classement stylistique - du moins lorsqu'elle se trouve dans un contexte artistique. Ainsi la critique a finalement dénoncé l'art conceptuel en tant qu'esthétique de bureaucratie ${ }^{2}$ (Benjamin Buchloh), voire « conceptual documentary style » pour citer Christian Kravagna ${ }^{3}$. Rétrospectivement on peut conclure qu'il y a un mouvement dialectique : dans l'histoire de la photographie, le style documentaire prend le relais par rapport au document photographique tandis que dans l'histoire de l'art, le document remplace le style pour se voir enfin récupéré par les rouages de l'histoire de styles. C'est cette constellation paradoxale au sujet de la photographie documentaire qui prépare l'avènement d'une photographie au second degré employant le document photographique au-delà des dichotomies habituelles telles que le style et l'information, le document et l'art.

1 Dan Graham dans : P. OSBORNE, Conceptual Art, Londres, Phaidon,2002, p. 134.

2 B. BUCHLOH, Conceptual Art 1962-1969. From the Aesthetic of Administration to the Critique of Institutions, in : S. PAGÉ (sous la dir. de), L'art conceptuel, une perspective, cat., Musée d'Art Moderne de la Ville de Paris, 1989, pp. 41-53.

3 C. KRAVAGNA, «It's nothing worth documenting if it's not difficult to get » : on the documentary nature of photography and film in the work of Gordon Matta-Clark, in : C. DISERENS (sous la dir. De), Gordon Matta-Clark, London, Phaidon, 2003, p. 138. 


\section{Appropriations - l'espace économique et institutionnel de l'art : Sherrie Levine}

Il n'est pas accidentel que Sherrie Levine, qui est l'une des premières à avoir réagi à l'aporie évoquée tantôt avec une stratégie d'appropriation, a consacré en 1981 toute une série au père du style documentaire, Walker Evans. En copiant quelques-unes des photos emblématiques du photographe, Levine introduit la figure de l'artiste comme plagiaire. Dans le sillage des discours postmodernistes, Sherrie Levine constate qu' "un tableau n'est qu'un espace dans lequel une variété d'images, toutes sans originalité, se fondent et s'entrechoquent"1. L'artiste déclare donc qu'on ne peut plus inventer quelque chose mais que la seule possibilité pour l'artiste est "d'imiter un geste qui est toujours antérieur, jamais original". Par conséquent, Levine photographie des images de Walker Evans depuis un catalogue d'exposition et les expose dans la Gallerie Metro Pictures à New York. En fait, cette démarche permet à Levine de dévoiler le paradoxe évoqué auparavant : faisant partie d'un grand projet mis en place par la Farm Security Administration (F.S.A.) dans les années 1930 pour documenter et améliorer les conditions de la population rurale aux ÉtatsUnis, les photos de Walker Evans sont devenues des icônes de la photographie artistique. Cependant, profitant du fait qu'au début des années 1980 ces images n'étaient plus soumises à un Copyright, Sherrie Levine put les reproduire et les exposer comme s'il s'agissait de ses propres œuvres. Selon ses propres termes, c'est la tension entre l'original et son œuvre qui l'intéresse. Afin de mettre en cause les relations entre reproduction et original, entre document multipliable et œuvre d'art unique, elle utilise plusieurs formats - de 4x5 inches jusqu'à $11 \times 14$ inches - et finit par limiter la dernière série dont les tirages sont numérotés ${ }^{2}$. Levine crée donc un double de la photographie d'Evans pour poser des questions sur l'authenticité de l'image photographique, sa fonction entre document et œuvre artistique ainsi que sur les droits de propriété qui définissent son statut économique et juridique. Cet aspect de re-reproduction qui est effectivement un état de second degré, est donc essentiel pour que l'image puisse dégager son potentiel de réflexion.

1 S. LEVINE, Déclaration (1982), in : C. HARRISON, P. WOOD (sous la dir.), Art en théorie, Paris, Hazan, 1997, p.. 1157.

2 Sherrie Levine dans un interview avec Constance Lewallen, in : Journal of Contemporary Art, $\mathrm{n}^{\circ}$ 6, 1993, version online : http://www.jca-online.com/slevine. html. 


\section{Rendre hommage aux 'morts' - l'espace de la mémoire : Christian Boltanski}

Les re-reproductions de l'artiste français Christian Boltanski fournissent la matière pour une réflexion sur la mémoire et la mort - deux notions récurrentes dans la théorie de la photographie quand il s'agit de caractériser les fonctions et les particularités du médium. Les monuments que Boltanski a réalisés en 1986 au sein de son installation à la Chapelle Saint Louis de l'hôpital La Salpêtrière à Paris, exposent la photographie au second degré en relation avec le lieu de présentation pour sonder l'espace de la mémoire. Les portraits photographiques proviennent d'une photographie de la classe de Boltanski de 1951 quand il avait sept ans. Après avoir re-photographié quelques visages, l'artiste les a agrandis et placés individuellement dans des cadres en fer. En alternant avec des images monochromes ces tableaux composent des configurations symétriques illuminées par des ampoules électriques. En procédant ainsi, Boltanski arrache les individus à un environnement naturel (probablement la cour d'école), de leur identité sociale (comme membres d'une classe) et de leur temporalité (les éléments qui pourraient indiquer le temps sont supprimés : vêtements, dates, textes, noms). Il les jette dans l'anonymat absolu, ce qui équivaut, selon l'artiste, à la mort : "de tous ces enfants, parmi lesquels je me trouvais et, très probablement, dont l'une des filles devait être celle que j'aimais, je ne me souvenais du nom d'aucun, je ne reconnaissais rien de plus que les visages sur la photographie. On aurait dit qu'ils avaient disparu de ma mémoire. On aurait dit que cette période était morte, puisqu'à présent, ces enfants devaient être des adultes dont je ne sais plus rien aujourd'hui. C'est pour cette raison que j'ai ressenti le besoin de rendre hommage à ces 'morts', qui, sur l'image, se ressemblent tous plus ou moins, comme des cadavres"1.

Comme des cadavres qu'il faut enterrer - pourrait-on ajouter. Cette association funéraire se manifeste sans équivoque dans un autre monument réalisé par l'artiste en 1996 pour une installation présentée au couvent de Santo Domingos de Bonaval à Saint-Jacques de Compostelle. $^{2}$

1 L. GUMPERT, Christian Boltanski, Paris, Flammarion, 1992, p. 83.

2 Voir: G. MOURE (sous la dir.), Christian Boltanski. Advent and other time, Centro Galego de Arte Contemporanea, Saint-Jacques de Compostelle, 1996. 
Comme les photographies des pierres tombales, les portraits de Boltanski sont des images qui prennent une autre signification par la décontextualisation. ${ }^{1}$ Mais tandis que les photographies funéraires ont la fonction de conserver la mémoire du défunt qui reste identifiable, les portraits de Boltanski sont condamnés à l'anonymat. Il ne s'agit pas d'images d'un individu mort mais d'images de la mort de l'individualité qui correspond à l'effacement absolu de la mémoire. Si chaque photographie est un agent de la mort - comme le suppose toute une phalange de critiques parmi lesquels Susan Sontag et Roland Barthes², pour ne citer qu'eux - l'installation de Boltanski est une réflexion sur la nature chimérique du médium susceptible de signifier à la fois, selon son contexte et son usage, la mémoire ainsi que l'oubli, la vie ainsi que la mort, le profane ainsi que le sacré.

\section{(Re)constructions - les espaces idéologiques des mass media : Thomas Demand}

L'artiste allemand Thomas Demand s'intéresse aux espaces idéologiques des mass média dans lesquels les photographies dites documentaires circulent. Salle de bain, par exemple, se réfère à l'un des scandales politiques les plus controversés de l'Allemagne de l'après-guerre. En 1987 le politicien Uwe Barschel a été retrouvé mort dans la baignoire de sa chambre de l'hôtel Beau-Rivage, à Genève. Suicide ou meurtre dissimulé ? L'enquête n'a pas apporté de réponse satisfaisante à la mort mystérieuse du politicien. La publication d'une photo sur la couverture du magazine Der Stern montrant le cadavre dans la baignoire a fait l'objet d'un débat virulent sur l'exploitation irrespectueuse de l'image du mort de la part des médias. Ainsi la photographie du lieu du crime provoqua une discussion ne concernant plus l'événement lui-même, mais sa diffusion par les médias. ${ }^{3}$ L'image se détache de son référent

1 Voir : A. HOLZER, Im Oval. Fotografien am Friedhof, in : Archive des Alltags, Heft 10, 1999, pp. 22-26.

2 Ainsi Roland BARTHES stipule dans La chambre claire que la photographie signifie toujours « un écrasement du Temps : cela est mort et cela va mourir. » (Gallimard, Le Seuil, 1980, p. 150) Susan SONTAG le pousse encore plus loin en disant que photographier signifie de dresser un inventaire de la mortalité (On Photography, London, Penguin Books, 2002, p 70).

3 C'est exactement ce processus d'autonomisation de la photographie auquel l'artiste s'intéresse : "If we consider the Barschel picture, it was the first reproduction that caused a big scandal. People started asking whether it should be printed at 
afin de créer sa propre réalité qui est soumise à ses qualités de médium ainsi qu'à son usage culturel, c'est-à-dire le programme de la technique et l'appareil de distribution ainsi que les conventions sociales et discursives qui définissent notre perception et compréhension du médium. ${ }^{1}$

Pour son œuvre Salle de bain, à partir de la photo bien connue du scandale Demand a fabriqué une maquette en papier, à échelle réelle, qu'il met en scène par un éclairage artificiel avant de la photographier. La maquette étant détruite après la prise, c'est la photographie sous diasec que l'artiste montre en guise d'œuvre d'art. Le spectateur se trouve donc devant une image brillante d'une architecture lisse et stérile, dépourvue de la moindre présence humaine. Ce qui semble être à première vue un instantané de la réalité révèle aussitôt son caractère artificiel par la monotonie du seul matériel utilisé, le carton, dont les bords effilés dévoilent le procédé de fabrication.

Ainsi la photographie de Demand nous démontre le caractère conventionnel et construit du médium par une double duperie : en simulant une réalité qui se dévoile aussitôt comme modèle artificiel, comme maquette de quelque chose, il sème un premier doute à l'égard de l'authenticité de l'objet représenté. En cachant l'origine de l'image les photographies ne sont accompagnées que des titres neutres et banals - Demand insinue un doute supplémentaire : la salle de bain apparaît comme un lieu aseptisé de la vie quotidienne ; en même temps la photo simule une image qui, par le biais des médias, s'est inscrite dans notre mémoire collective. Une photographie de Demand éveille toujours le soupçon du déjà-vu, mais de façon différente. Ce malaise qui ressort de la tension entre la banalité du sujet, la stérilité froide de la maquette et le sentiment du déjà-vu atteint une nouvelle dimension au moment de la

all. [They asked] [w]ether a photographer should be allowed to intrude and take a picture before the police had arrived. Much later, the untiring public prosecutor in Schleswig-Holstein reexamined the unresolved questions on the basis of the photos. That means, the photo medium runs through the whole affair. »In : R. MARCOCI (sous la dir.), Thomas Demand, Catalogue, Museum of Modern Art New York, New York 2005, pp. 20-21.

1 L'hypothèse de la détermination de la pratique et de la réception de l'image photographique par l'appareil et le programme du médium a été énoncée par le philosophe Vilém Flusser (V. FLUSSER, Pour une théorie sur la photographie, Paris, Circe Editions 1996). Au début des années 1980, l'artiste et théoricien Victor Burgin fut l'un des premiers à exiger l'analyse de la photographie en tant que «phénomène culturel général » dont la signification jaillit des discours socioculturels. V. BURGIN, Thinking Photography, London and Basingstoke, The Macmillan Press Ltd., 1982. 
découverte de la source d'image. Tout d'un coup l'image se charge des souvenirs qui, pourtant, ne se réfèrent pas à l'événement même, mais aux images et aux informations transmises par les médias gravées dans la mémoire.

Demand constate que le sentiment d'authenticité fonctionne selon des règles précises. "Il s'agit d'une convention de la société, un code, qui est essentiel pour la photographie de presse en général. Et c'est cela qui m'intéresse : le code, qui existe comme convention tout à fait indépendamment du lieu réel"'. En opérant dans la brèche entre l'objet et la maquette, l'événement et sa représentation, Demand cherche à dépister les mécanismes de la constitution d'un monde virtuel par les médias et les conventions culturelles qui créent le sentiment d'authenticité ressenti devant une photographie. Bien qu'il ne s'agisse pas d'une photographie au second degré dans le sens littéral du terme (la maquette constituant une étape intermédiaire), le principe reste le même : la photographie remonte à une photographie antérieure dont les paramètres essentiels - tels que le cadrage et la situation dans le champ - sont maintenus. Ainsi se dégage une multiplication vertigineuse de représentations qui passe par la photographie de presse, la maquette de la photographie « originale » et, enfin, la photographie finale. Cette réaction en chaîne de représentations a été assimilée, à juste titre, à la théorie du constructivisme radical de Ernst von Glaserfeld. Selon le psychologue cognitif “nous n'avons pas d'accès au monde 'réel' sinon par le biais de nos expériences et d'un acte de la connaissance, ce qui, d'après la définition, ne peut aboutir à rien d'autre qu'à une autre représentation"2.

\section{L'entre-deux - L'espace virtuel des média : David Claerbout}

De même que Demand, quoique sous un autre angle de vue, l'artiste belge David Claerbout trouble l'idée de l'authenticité et de la véracité de la photographie. Plutôt que de se pencher sur les stratégies et les effets des mass média, Claerbout s'empare des nouvelles possibilités de l'image numérique pour saper les conditions de base des photos et du film. En fait, en introduisant du mouvement dans des photos existantes,

1 Interview avec Ruedi Widmer, in : R. MARCOCI, op. cit., p. 11 (traduction de l'auteur).

2 E. VON GLASERFELD, Radikaler Konstruktivismus: Ideen, Ergebnisse, Probleme, Frankfurt a. M., Suhrkamp, p.. 158 (traduction de l'auteur). 
Claerbout met à l'épreuve la frontière traditionnellement établie entre l'image fixe de la photographie et l'image mobile du film.

Provenant d'un des innombrables ouvrages ou catalogues que l'artiste utilise comme sources d'image et d'inspiration, la photo utilisée dans Shadow Piece date des années cinquante ${ }^{1}$. Cette photo nous montre le hall d'entrée d'un grand bâtiment publique dont les portes en verre donnent sur le trottoir ensoleillé. Le point de vue du spectateur est déterminé par l'œil de l'appareil photo, positionné au premier étage. Il y a ici un mouvement oscillant entre inclusion et exclusion qui concerne d'abord le rapport entre l'image et le spectateur : mis à l'écart par la balustrade, le spectateur se trouve manifestement en dehors du champ pictural, si bien que l'escalier sous voûte l'entraîne inévitablement vers le rez-de-chaussée. Cependant, le statisme de la photographie s'anime brusquement lorsque des personnes entrent en scène et font mine de vouloir franchir les portes en verre. En vain, parce que celles-ci restent fermées. Cette fois le jeu entre inclusion et exclusion vise la réalité inhérente à l'image. Le monde représenté par la photographie reste interdit aux personnages de la vidéo, copiés postérieurement dans l'image. Cette interdiction est historique - notons le décalage qui sépare le moment de la prise de la photo et celui où les personnages ont été filmés ; mais aussi et surtout médiatique : la barrière invisible s'érige entre le monde de la photographie et celui du film - de l'image fixe et de l'image mobile.

Pourtant, cette double exclusion est parfaitement dissimulée : les personnages portent des vêtements des années 50, la couture entre l'image photographique et la vidéo n'est presque plus visible. De plus, les ombres semblent mettre en place l'illusion parfaite d'un espace transréel réunissant les mondes virtuels de la photographie et du film ainsi que celui du spectateur. Cette superposition de réalités différentes engendre un effet d'immersion, car le spectateur est plongé dans l'image grâce aux dimensions de l'écran, le mouvement de la balustrade et, enfin, le croisement de son ombre avec celles de l'architecture photographiée et des personnes filmées. Pourtant, ce sont les ombres qui dévoilent la supercherie du scénario. En fait, la qualité tonale de l'ombre change en fonction des trois espaces différents constituant l'œuvre : les espaces

1 Dans une conversation en mai 2007, l'artiste m'a indiqué avoir trouvé la photographie dans un catalogue sur l'architecture moderne dont il aurait oublié la référence exacte. Apparemment, ce n'est pas le caractère documentaire de la photo qui l'intéresse, mais son effet spatial et temporel. 
virtuels du film et de la photo ainsi que l'espace réel du spectateur. En fin de compte, ce jeu complexe d'exclusion et d'inclusion sur les niveaux narratif, temporel, spatial et médial met à l'épreuve les stratégies d'immersion et d'illusion qui soutiennent les mythes de l'authenticité et de la crédibilité de la photographie et du film.

La photographie au second degré ouvre plusieurs pistes de réflexion afin de reconsidérer des dichotomies régulièrement tracées dans les discours sur la photographie, comme celles qui sépare document et art, information et style. La structure complexe de ces chimères photographiques peut être illustrée par la conjonction de deux modèles sémiologiques - le système sémiologique étant responsable selon Roland Barthes pour la construction du mythe et la théorie d'indices proposée par Henri van Lier.

Dans ses Mythologies, Roland Barthes désigne un schéma pour illustrer le fonctionnement du mythe ${ }^{1}$. Selon lui le mythe contient deux systèmes sémiologiques dont le premier, le langage-objet, se réfère à ce qu'on appelle le référent, tandis que le second, le méta-langage, se greffe sur le langage-objet pour construire son propre système - le mythe. Ainsi le soldat noir faisant le salut militaire français devant le tricolore nous indique les principes de l'égalité (tous les français, sans distinction de couleur, servent sous le drapeau tricolore) et la gloire de l'empire, tout en corroborant une image positive du colonialisme (le zèle du noir devant le drapeau). Il y a donc duplication de la structure sémiotique et déplacement au niveau du message ou, plus précisément, du référent. Or, si l'on accepte avec Henri van Lier que le caractère spécifique de la photographie réside dans la relation entre index et indice, on constate les mêmes mécanismes de duplication et de déplacement dans la photographie au second degré. Selon van Lier les index (cadrage, situation dans le champ, éclaircissement, etc.) accentuent ou orientent les indices photographiques, les empreintes des photons, qui "renvoient à leur cause (à leur spectacle éventuel) et par monstration et par démonstration"2.

Par conséquent, la photographie au second degré constitue une meta-image par le dédoublement du référent et le déplacement de l'orientation indexicale. Dédoublement, parce qu'à l'image antérieure

1 R. BARTHES, Mythologies, Paris, Seuil 1957, p. 222.

2 H. VAN LIER, Philosophie de la Photographie, Bruxelles, Les Impressions Nouvelles, 2005 (1 ère éd. 1983), p. 25. 
s'ajoute une nouvelle image sans que la première disparaisse - elle reste présente par transparence dans le sens où Genette donne au palimpseste. Déplacement, car les indices ne désignent plus le spectacle que la photo donne à voir mais une autre photographie. Là réside le côté pervers de la photographie au second degré qui n'est rien d'autre qu'une empreinte d'une empreinte. Pourtant, il faut bien distinguer la structure et surtout l'intention du mythe de la photographie au second degré. Tandis que le mythe tente de dissimuler la tension entre les deux systèmes sémiologiques afin de mieux embobiner le receveur, la photographie au second degré fait remarquer la tension existant entre image et référent, original et reproduction.

La perte de contact avec la « vraie » réalité est donc nécessaire pour mettre à l'épreuve la relation qui s'instaure et se restaure sans cesse entre l'image photographique et ses désignés, ses référents ou encore son spectacle signalé (Henri van Lier) en-deçà et au-delà de la scission ontologique ente document et art, ou bien entre information et style. Mais il ne faut pas oublier que du fond de cette forme de maïeutique picturale, un inassouvissable plaisir de l'image jaillit dans les stratégies de reproduction, de réappropriation, de recyclage et de transformation permettant de récréer à l'infini l'imagerie de notre monde. Autrement dit, l'image au second degré n'est qu'un des noms de cette incessante circulation des images sans quoi la photographie ne vaudrait pas un seul regard. Et quand je dis un regard... 
